\title{
Hemoglobin, albumin, lymphocytes and platelets (HALP) score is a useful predictor of survival in patients with recurrent glioblastoma multiforme treated with bevacizumab plus irinotecan
}

\section{Mustafa Korkmaz ( $\sim$ dr.musstafa@gmail.com )}

Necmettin Erbakan University Meram Faculty of Medicine: Necmettin Erbakan Universitesi Meram Tip Fakultesi https://orcid.org/0000-0003-0926-6748

\section{Melek Karakurt Eryılmaz}

Necmettin Erbakan University Meram Faculty of Medicine: Necmettin Erbakan Universitesi Meram Tip Fakultesi

\section{Mehmet zahid koçak}

Necmettin Erbakan University Meram Faculty of Medicine: Necmettin Erbakan Universitesi Meram Tip Fakultesi

\section{Aykut Demirkıran}

Necmettin Erbakan University Meram Faculty of Medicine: Necmettin Erbakan Universitesi Meram Tip Fakultesi

\section{mustafa Karaağaç}

Necmettin Erbakan University Meram Faculty of Medicine: Necmettin Erbakan Universitesi Meram Tip Fakultesi

\section{murat araz}

Necmettin Erbakan University Meram Faculty of Medicine: Necmettin Erbakan Universitesi Meram Tip Fakultesi

\section{Mehmet Artaç}

Necmettin Erbakan University Meram Faculty of Medicine: Necmettin Erbakan Universitesi Meram Tip Fakultesi

\section{Research Article}

Keywords: Bevacizumab, Glioblastoma multiforme, HALP, Predictive marker

Posted Date: December 27th, 2021

DOI: https://doi.org/10.21203/rs.3.rs-809597/v2 
License: (c) (i) This work is licensed under a Creative Commons Attribution 4.0 International License. Read Full License 


\section{Abstract}

Backgrounds: We aimed to investigate whether the HALP score is a predictive marker in patients with recurrent GBM who were given bevacizumab plus irinotecan.

Methods: We compared the survival of patients followed up in our clinic with the diagnosis of recurrent GBM and treated with bevacizumab plus irinotecan, according to HALP score.

Results: Median PFS and OS were 4.5 (0.9-14.9) and 8 (0.9-21.3) months, respectively. The median PFS of the low HALP score group was 1.85 (1.3-3.37) months, and of the high HALP score group was 4.96 (0.9-14.9) months $(p=0.03)$. The OS of the high HALP score group (9.63 [7.28-11.9]) was statistically higher compared with low HALP score group $(2.26$ [0.88-3.65]) $(\mathrm{p}<0.001)$. In univariate analysis HALP score was a significant prognostic factor; patients with low HALP score had a poorer prognosis than high HALP score (HR: 0.063, $p<0.001)$. The multivariate analysis showed that HALP score $(p=0.003)$, and residual tumor $(\mathrm{p}=0.029)$ were significant prognostic factors. In multivariate Cox regression analysis, low HALP score was a significant poor prognostic factor for OS compared with high HALP score (HR: 0.063, $p<0.001)$.

Conclusion: We showed that the HALP score at the start of treatment is an independent prognostic factor for PFS and OS in patients with recurrent GBM treated with bevacizumab plus irinotecan. The HALP score, which can be easily calculated by routine tests before chemotherapy, can be used as a predictive marker for bevacizumab treatment decision.

\section{Introduction}

Glioblastoma multiforme (GBM) is the most common malignant primary brain tumor in adults. The standard treatment approach is the combination of adjuvant radiation therapy (RT) with concurrent and adjuvant temozolomide following surgery. The median overall survival (OS) is 15 months, and even with the most appropriate treatment, most patients die within two years [1, 2].

GBM is a highly vascularized tumor that requires extensive collection of blood vessels to combat hypoxia. Vascular endothelial growth factor (VEGF)-mediated inhibition of angiogenesis by bevacizumab is a therapeutic strategy for GBM at the center of several clinical studies [3]. A meta-analysis of 14 clinical studies showed that bevacizumab did not improve OS, but improved radiographic response rates and progression-free survival (PFS) in GBM, either as a single agent or in combination with chemotherapy [4]. And it is currently only offered as salvage therapy for treatment-resistant cases. It has been shown that radiological imaging, clinical and laboratory parameters predict bevacizumab response in recurrent GBM [5-7].

Hemoglobin, albumin, lymphocyte, and platelet (HALP) score has been shown to be a new and potential prognostic indicator for various malignancies [8-17]. We aimed to investigate whether the HALP score is a predictive marker in patients with recurrent GBM who were given bevacizumab plus irinotecan. 


\section{Methods}

Thirty-one patients with a pathologically confirmed diagnosis of GBM treated with bevacizumab plus irinotecan at first line treatment, were included in the study. The study protocol was approved by the local ethics committee. Medical database of GBM subjects who were admitted to out-patient clinics of our institution between April 2015 and July 2019, has been retrospectively analyzed. Patients who did not receive at least one cycle of bevacizumab plus irinotecan were not included in the study. Since Isocitrate dehydrogenase (IDH) mutation, 1p/19q co-deletion, 06-methylguanine-DNA methyltransferase (MGMT) could not be studied in our center, analysis could not be performed on these. Albumin and hemogram levels before receiving treatment were recorded from the electronic files of the patients. The HALP score was calculated according to the formula: hemoglobin $(\mathrm{HB})(\mathrm{g} / \mathrm{L}) \times$ albumin $(\mathrm{g} / \mathrm{L})$ x lymphocytes (/L)/platelets (/L). PFS was defined as the time from the initiation of bevacizumab plus irinotecan to the first radiological progression, while OS was defined as the time from the initiation of therapy to death from any cause.

\section{Statistical Analysis}

The optimal cut-off value of the HALP score was analyzed using X-tile software version 3.6.1 (Yale University, New Haven CT, USA). Kaplan-Meier method was used for survival analysis with the log-rank test used to statistical difference. A univariate Cox proportional hazards regression model was used to evaluate the prognostic value of each variable for OS. Multivariate Cox proportional hazards regression models were used to analyze independent prognostic factors. Homogenous variables in study groups according the HALP score were compared by independent samples t-test and expressed as mean \pm standard deviation. Non-homogenous variables were compared by Mann-Whitney $\mathrm{U}$ test and expressed as median (minimum-maximum). Chi-square test or Fisher exact test was performed to comparison of categorical variables. A p value of $<0.05$ is considered as statistically significant. Data were analyzed by SPSS software. (SPSS 15.0; IBM Inc., Chicago, IL, USA).

\section{Results}

Thirty-one patients were included the study, $5(16 \%)$ were female and $26(84 \%)$ were male. The mean age of all subjects was $46.3 \pm 12.2$ years. The Eastern Cooperative Oncology Group Performance Status (ECOG-PS) of $22(71 \%)$ patients was 1, and that of $9(29 \%)$ patients was 2. Median follow-up time was 7.86 (0.33-21.3) months. 27 (87.1\%) patients had received adjuvant temozolomide. Laboratory parameters were as follows; HB: 14.09 (11.2-18.6) g/dl, albumin 39.1 (29-49) g/L, lymphocyte 1.2 (0.22.70) $10^{3} / \mu \mathrm{L}$, platelet 229 (79-385) $10^{9} / \mathrm{L}$. Median PFS and OS were $4.5(0.9-14.9)$ and 8 (0.9-21.3) months, respectively. The cut-off value for the HALP score for OS was 18 in analysis with using X-tile software (figure 1). Those with a HALP score of 18 and below were defined as the low HALP score group, and those above 18 were defined as the high HALP score group. Of the 31 patients, 13 had a low HALP score and 18 had a high HALP score. Age, gender, and ECOG-PS were not statistically different between the two groups (low and high HALP score group) ( $p=0.76, p=0.1,-p=0.12$, respectively). There was no 
statistical difference between the histological type, residual tumor, tumor location, and surgery type low and high HALP score groups ( $p>0.05$ for all, Table 1 ). The median PFS of the low HALP score group was 1.85 (1.3-3.37) months, and of the high HALP score group was 4.96 (0.9-14.9) months. This difference was statistically significant $(p=0.03)$. The OS of the high HALP score group (9.63 [7.28-11.9]) was statistically higher compared with low HALP score group $(2.26$ [0.88-3.65]) $(p<0.001)$.

Univariate analysis showed that HALP Score ( $\leq 18$ vs $>18$ ), residual tumor (no vs. yes), and ECOG-PS (1 vs. 2) were important prognostic factors (Table 2). HALP Score was a significant prognostic factor; patients with low HALP score had a poorer prognosis than high HALP score ( $\leq 18$ vs. $>18$; HR: $0.063,95 \%$ Cl: $0.016-0.249, p<0.001)$. The multivariate analysis showed that HALP score $(p=0.003)$, and residual tumor ( $p=0.029$ ) were significant prognostic factors. In multivariate Cox regression analysis, low HALP score was a significant poor prognostic factor for OS compared with high HALP score ( $\leq 18$ vs. $>18$; HR: 0.063, 95\% Cl: 0.016-0.249, $p<0.001$ ) (Table 2).

\section{Discussion}

We found that the HALP score at the beginning of treatment in recurrent GBM patients treated with bevacizumab plus irinotecan is an independent prognostic factor for PFS and OS. PFS and OS were significantly shorter for patients in the low HALP score group than those in the high HALP score group.

Inflammatory factors have been recognized as an important contributing factor to the complexity and lethality of GBM. Therefore, many studies are carried out considering that inflammatory factors may be prognostic factors for cancers, and the search continues. Previous studies have reported that tumor necrosis factor alpha (TNF- $\alpha$ ) and C-reactive protein (CRP) are noticeably higher in patients with glioblastoma compared to healthy controls. However, the relationship between inflammatory factors and glioma risk or prognosis is controversial. There are positive and negative studies on this [18]. A recent study showed that elevations in all inflammatory markers were associated with poor OS in those using bevacizumab in recurrent GBM, but only elevated CRP to albumin ratio (CAR) and platelet to lymphocyte ratio (PLR) were reported to be an independent predictive factor. The prognostic significance of the increase in neutrophil to lymphocyte ratio (NLR) has not been demonstrated in this study [19].

As known, while hemoglobin and albumin levels are associated with the nutrition status of the body, lymphocytes and platelets are related to the immune system. The HALP score has been usually used to predict the prognosis of some types of cancers by using the character of showing the two main roles (inflammation and nutrition status) in the prognosis of cancer [16]. Chen et al. firstly described this HALP score to predict the prognosis of gastric cancer. In 1332 patients with gastric cancer who underwent gastrectomy, the preoperative HALP $\geq 56.8$ group had a significantly better prognosis than the HALP < 56.8 group [9]. Also, the prognostic effectiveness of the HALP score has been investigated in; esophageal squamous cell cancer [10], pancreatic cancer [11], colorectal cancer [12], renal cell carcinoma [13], bladder cancer [14], prostate cancer [15, 16], and small cell lung cancer $[17,18]$. Low HALP score was associated 
with worse survival outcomes in all studied cancers. However, the optimum cut-off value was different in each study, so it is very important to define the optimal cut-off value of HALP score.

Bevacizumab, a humanized monoclonal IgG1 antibody, acts to bind to human VEGF and inhibit its activity [20]. The objective response rate (ORR) achieved with the combination of bevacizumab plus irinotecan in recurrent GBM is 38\%, and the median PFS and OS are 5.6 and 9.2 months, respectively [21]. Predictive factors of bevacizumab response in recurrent GBM have been investigated in several studies. In these studies, it has been shown that the development of hypertension and proteinuria, high eosinophil and lymphocyte levels, and low CAR and PLR values due to bevacizumab treatment are predictive for better survival $[6,7,19]$.

In conclusion, this is the first study to show that the HALP score predicts the response to bevacizumab plus irinotecan treatment in patients with recurrent GBM. This score will be useful because it can be easily calculated with routine blood tests, is cheap and objective. We think that it can be used in routine practice if the same result is obtained as a result of studies with a larger number of patients.

\section{Declarations}

Funding: Nil

Conflicts of interest/Competing interests: All authors state that they have no conflict of interests to declare

Funding: This research did not receive any specific grant from funding agencies in the public, commercial, or not-for-profit sectors

Conflicts of interest/Competing interests: None

Ethics approval: The study protocol was approved by the Necmettin ErbakanUniversity Faculty of Medicine ethics committee (Approval no:2020/2749).

\section{References}

1. Stupp R, Mason WP, van den Bent MJ, Weller M, Fisher B, et al; European Organisation for Research and Treatment of Cancer Brain Tumor and Radiotherapy Groups; National Cancer Institute of Canada Clinical Trials Group. Radiotherapy plus concomitant and adjuvant temozolomide for glioblastoma. N Engl J Med. 2005 Mar 10;352(10):987-996. doi: 10.1056/NEJMoa043330.

2. Stupp R, Hegi ME, Mason WP, van den Bent MJ, Taphoorn MJ, et al; European Organisation for Research and Treatment of Cancer Brain Tumour and Radiation Oncology Groups; National Cancer Institute of Canada Clinical Trials Group. Effects of radiotherapy with concomitant and adjuvant temozolomide versus radiotherapy alone on survival in glioblastoma in a randomised phase III study: 
5-year analysis of the EORTC-NCIC trial. Lancet Oncol. 2009 May;10(5):459-466. doi: 10.1016/S14702045(09)70025-7.

3. Hundsberger T, Reardon DA, Wen PY. Angiogenesis inhibitors in tackling recurrent glioblastoma. Expert Rev Anticancer Ther. 2017 Jun;17(6):507-515. doi: 10.1080/14737140.2017.1322903.

4. Kaka N, Hafazalla K, Samawi H, Simpkin A, Perry J, et al. Progression-Free but No Overall Survival Benefit for Adult Patients with Bevacizumab Therapy for the Treatment of Newly Diagnosed Glioblastoma: A Systematic Review and Meta-Analysis. Cancers (Basel). 2019 Nov 4;11(11):1723. doi: 10.3390/cancers11111723.

5. Kickingereder P, Wiestler B, Burth S, Wick A, Nowosielski M, et al. Relative cerebral blood volume is a potential predictive imaging biomarker of bevacizumab efficacy in recurrent glioblastoma. Neuro Oncol. 2015 Aug;17(8):1139-1147. doi: 10.1093/neuonc/nov028.

6. Carvalho B, Lopes RG, Linhares P, Costa A, Caeiro C, et al. Hypertension and proteinuria as clinical biomarkers of response to bevacizumab in glioblastoma patients. J Neurooncol. 2020 Mar;147(1):109-116. doi: 10.1007/s11060-020-03404-z.

7. Vaios EJ, Winter SF, Muzikansky A, Nahed BV, Dietrich J. Eosinophil and lymphocyte counts predict bevacizumab response and survival in recurrent glioblastoma. Neurooncol Adv. 2020 Mar 11;2(1):vdaa031. doi: 10.1093/noajnl/vdaa031.

8. Chen XL, Xue L, Wang W, Chen HN, Zhang WH, et al. Prognostic significance of the combination of preoperative hemoglobin, albumin, lymphocyte and platelet in patients with gastric carcinoma: a retrospective cohort study. Oncotarget. 2015 Dec 1;6(38):41370-41382. doi: 10.18632/oncotarget.5629.

9. Hu SJ, Zhao XK, Song X, Lei LL, Han WL, et al. Preoperative maximal voluntary ventilation, hemoglobin, albumin, lymphocytes and platelets predict postoperative survival in esophageal squamous cell carcinoma. World J Gastroenterol. 2021 Jan 28;27(4):321-335. doi: 10.3748/wjg.v27.i4.321.

10. Xu SS, Li S, Xu HX, Li H, Wu CT, et al. Haemoglobin, albumin, lymphocyte and platelet predicts postoperative survival in pancreatic cancer. World J Gastroenterol. 2020 Feb 28;26(8):828-838. doi: 10.3748/wjg.v26.i8.828.

11. Jiang H, Li H, Li A, Tang E, Xu D, et al. Preoperative combined hemoglobin, albumin, lymphocyte and platelet levels predict survival in patients with locally advanced colorectal cancer. Oncotarget. 2016 Nov 1;7(44):72076-72083. doi: 10.18632/oncotarget.12271.

12. Peng D, Zhang CJ, Tang Q, Zhang L, Yang KW, et al. Prognostic significance of the combination of preoperative hemoglobin and albumin levels and lymphocyte and platelet counts (HALP) in patients with renal cell carcinoma after nephrectomy. BMC Urol. 2018 Mar 15;18(1):20. doi: 10.1186/s12894018-0333-8.

13. Peng, D., Zhang, Cj., Gong, Yq. Hao H., Guan B. et al. Prognostic significance of HALP (hemoglobin, albumin, lymphocyte and platelet) in patients with bladder cancer after radical cystectomy. Sci Rep 8, 794 (2018). https:// doi.org/10.1038/s41598-018-19146-y. 
14. Guo Y, Shi D, Zhang J, Mao S, Wang L, et al. The Hemoglobin, Albumin, Lymphocyte, and Platelet (HALP) Score is a Novel Significant Prognostic Factor for Patients with Metastatic Prostate Cancer Undergoing Cytoreductive Radical Prostatectomy. J Cancer. 2019 Jan 1;10(1):81-91. doi: 10.7150/jca.27210.

15. Kaya C, Caliskan S, Sungur M, Aydın C. HALP score and albumin levels in men with prostate cancer and benign prostate hyperplasia. Int J Clin Pract. 2021 Mar;75(3):e13766. doi: 10.1111/ijcp.13766.

16. Shen XB, Zhang YX, Wang W, Pan YY. The Hemoglobin, Albumin, Lymphocyte, and Platelet (HALP) Score in Patients with Small Cell Lung Cancer Before First-Line Treatment with Etoposide and Progression-Free Survival. Med Sci Monit. 2019 Jul 29;25:5630-5639. doi: 10.12659/MSM.917968.

17. Yang N, Han X, Yu J, Shu W, Qiu F, et al. Hemoglobin, albumin, lymphocyte, and platelet score and neutrophil-to-lymphocyte ratio are novel significant prognostic factors for patients with small-cell lung cancer undergoing chemotherapy. J Cancer Res Ther. 2020 Sep;16(5):1134-1139. doi: 10.4103/jcrt.JCRT_1066_19.

18. Feng $Y$, Wang J, Tan D, Cheng P, Wu A. Relationship between circulating inflammatory factors and glioma risk and prognosis: A meta-analysis. Cancer Med. 2019 Dec;8(17):7454-7468. doi: 10.1002/cam4.2585.

19. Besiroglu M, Shbair AT, Yasin Al, Topcu A, Turk HM, et al. Systemic Inflammatory Markers for Prediction of Bevacizumab Benefit in Glioblastoma Multiforme. J Coll Physicians Surg Pak. 2021 Jan;31(1):39-44. doi: 10.29271/jcpsp.2021.01.39.

20. Ghiaseddin A, Peters KB. Use of bevacizumab in recurrent glioblastoma. CNS Oncol. 2015;4(3):15769. doi: $10.2217 / \mathrm{cns} .15 .8$.

21. Friedman HS, Prados MD, Wen PY, Mikkelsen T, Schiff D, et al. Bevacizumab alone and in combination with irinotecan in recurrent glioblastoma. J Clin Oncol. 2009 Oct 1;27(28):4733-40. doi: 10.1200/JCO.2008.19.8721.

\section{Tables}

Table 1: Demographic and clinical characteristics of the patients according to the HALP score. (HALP: hemoglobin, albumin, lymphocyte, and platelet) 


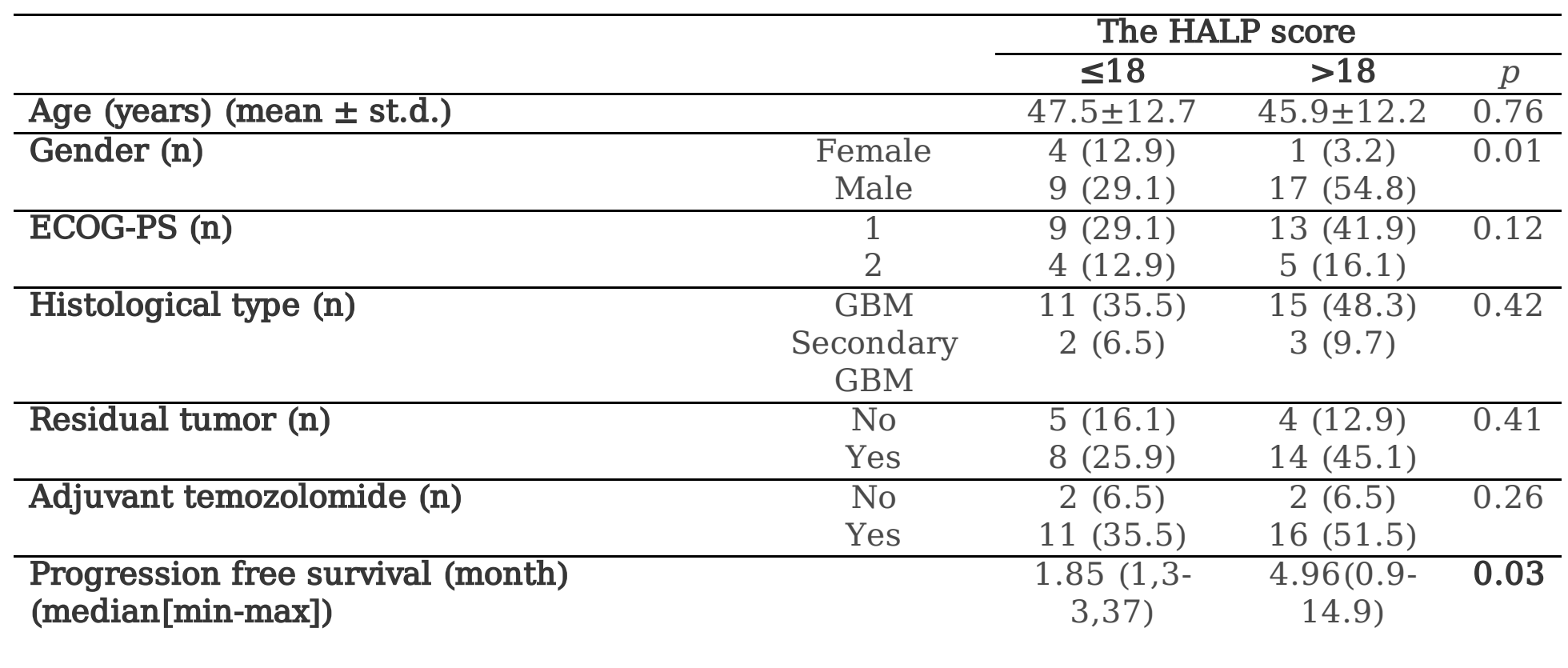

ECOG-PS: The Eastern Cooperative Oncology Group Performance Status. GBM: Glioblastoma multiforme.

Table 2: Univariate and Multivariate analyses of overall survival in patient with brain tumor.

\begin{tabular}{|l|l|l|l|l|l|l|}
\hline & \multicolumn{3}{|l|}{ Univariate analyses } & \multicolumn{3}{l|}{ Multivariate analyses } \\
\hline Variable & HR & $\mathbf{9 5 \%} \mathbf{C l}$ & $\mathbf{p}$ & HR & $\mathbf{9 5 \% ~ C l}$ & $\mathbf{p}$ \\
\hline HALP Score $(\leq 18$ vs $>18)$ & 0.063 & $0.016-0.249$ & $\mathbf{< 0 . 0 0 1}$ & 0.077 & $0.015-0.408$ & $\mathbf{0 . 0 0 3}$ \\
\hline Residual tumor (No vs Yes) & 3.445 & $1.449-8.192$ & $\mathbf{0 . 0 1 5}$ & 2.69 & $0.222-3.646$ & $\mathbf{0 . 0 2 9}$ \\
\hline Gender (F vs M) & 0.414 & $0.151-1.164$ & 0.08 & 0.89 & $0.222-3.646$ & 0.88 \\
\hline ECOG-PS (1 vs 2) & 3.07 & $1.230-7.680$ & $\mathbf{0 . 0 1 6}$ & 2.14 & $0.841-5.492$ & 0.11 \\
\hline
\end{tabular}

F: female; M: male; HALP - hemoglobin, albumin, lymphocyte, and platelet; HR - hazard ratio; CI - confidence interval.

\section{Figures}



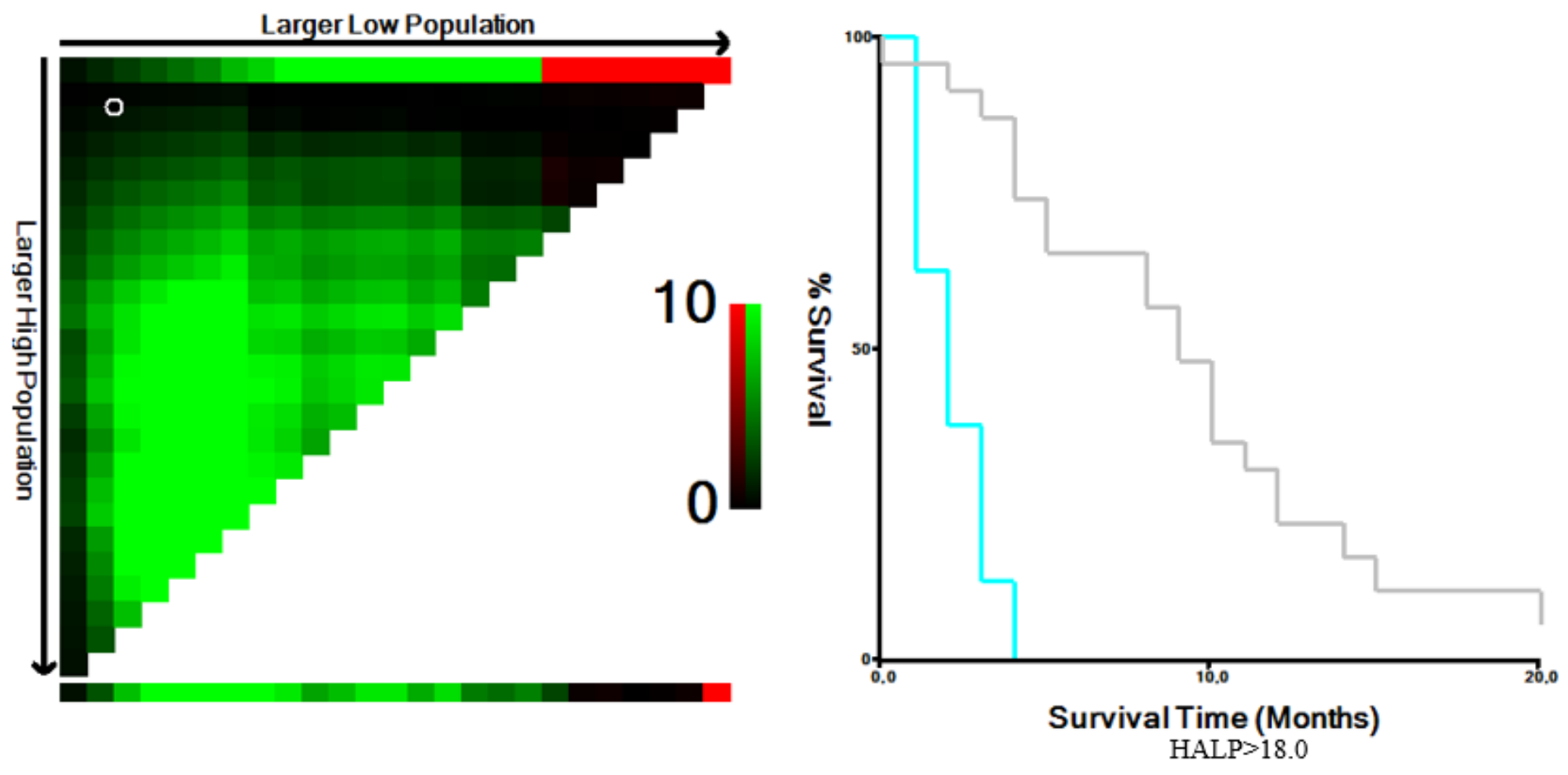

HALP $>18.0$

Figure 1

Cut- off values for the HALP score (hemoglobin, albumin, lymphocyte, and platelet) by X-tile software version 3.6.1. 